\title{
Yersiniosis in children
}

\author{
MARKKU MÄKI, TIMO VESIKARI, IMMO RANTALA, AND PAUL GRÖNROOS
}

Department of Paediatrics and Department of Microbiology, Tampere Central Hospital, and Institute of Clinical Sciences, University of Tampere, Finland

SUMMARY 40 cases of bacteriologically proved Yersinia enterocolitica infections in children under 15 years were reviewed. Most children presented with abdominal symptoms, and diarrhoea was present in $80 \%$ of them. In half of those with diarrhoea the stools were mucoid and gross blood was often present. Faecal leucocytes were found in 4 out of the 5 children studied. The clinical findings are consistent with the enteroinvasive pathogenic mechanism proposed for $Y$. enterocolitica. 29 of 30 faecal isolates of $Y$. enterocolitica were found invasive for human epithelial cells in vitro. Nine strains produced an enterotoxin demonstrable in newborn mouse assay. Toxin production may be an additional pathogenic mechanism in human yersiniosis.

Yersinia enterocolitica has been increasingly recognised as a human pathogen during the last few years. In adults $Y$. enterocolitica infections are mainly due to secondary immunological complications, particularly arthritis and erythema nodosum, ${ }^{1-3}$ which are rare in children. In fact, yersiniosis in small children has been reported to be a relatively mild infection causing diarrhoeal disease not clinically different from other forms of acute gastroenteritis. ${ }^{4}$ However, chronic diarrhoea has also been described. ${ }^{5}$ In older children $Y$. enterocolitica infection has often been associated with mesenterial lymphadenitis causing an acute, appendicitis-like disease. ${ }^{6}$

It has recently been shown that $Y$. enterocolitica from human sources is invasive for human epithelial cells in vitro. ${ }^{7-9}$ We have demonstrated polymorphonuclear leucocytes in the stools of patients with $Y$. enterocolitica infection, and suggested an association between the invasiveness of the pathogen, clinical exudative diarrhoea, and the presence of faecal leucocytes. ${ }^{9}$

We have now extended our studies on child yersiniosis by analysing clinical features in $\mathbf{4 0}$ cases of bacteriologically proved $Y$. enterocolitica infection. It appears that the present data lend further support to the concept that yersiniosis in children is frequently associated with exudative diarrhoea and signs of systemic disease, both consistent with an invasive pathogen. We now present further evidence of the intracellular invasiveness of $Y$. enterocolitica. At the same time we have made observations of 'heat-stable like' toxin (ST) production, which we believe may be an additional pathogenic mechanism in $Y$. enterocolitica infections.

\section{Methods}

Patients. 40 isolations of $Y$. enterocolitica from faeces of children under 15 years of age were recorded during the period from December 1974 to June 1978. Five patients were studied as part of a project on gastroenteritis in children, and in these children faecal leucocytes were searched for. In the remaining cases (before May 1977) the clinical symptoms and findings were retrospectively collected from medical records.

Laboratory studies. $Y$. enterocolitica was isolated from faeces using a modified Rappaport enrichment medium or the direct plating method of Wauters, ${ }^{10}$ or sometimes both. The biotypes of $Y$. enterocolitica were determined according to Wauters's classification. ${ }^{11}$ The isolates were serotyped by a slide agglutination method using specific rabbit antisera against serotypes $0: 3$ and $0: 9 .{ }^{12}$

Antibiotic sensitivity was determined by the semiquantitative disc diffusion method. ${ }^{13}$ The results are expressed, using the new Scandinavian division, as falling into 3 sensitivity groups: sensitive, intermediate, or resistant. ${ }^{14}$

The invasiveness test in HEp-2 cell culture was done as described previously. ${ }^{9}$ For electron microscopical examination, the cells were fixed in a mixture of $2 \%$ paraformaldehyde and $2 \%$ glutaraldehyde in $0.1 \mathrm{~mol} / 1$ phosphate buffer at $\mathrm{pH} 7 \cdot 4$. 
After postfixation in $2 \%$ osmium tetroxide, the cells were dehydrated through a series of acetones and embedded in Epon 812. Thin sections were stained with lead citrate and uranyl acetate.

In addition to HEp-2 cell cultures, 12 isolates of $Y$. enterocolitica were also tested for invasiveness in the guinea-pig conjunctiva model as described by Serény. ${ }^{15}$

Bacteria-free culture supernatants produced at room temperature, as described by Pai and Mors, ${ }^{16}$ were tested for enterotoxin activity in infant mice, ${ }^{17}$ and in Y-1 adrenal cell cultures, ${ }^{18}$ including ST and heat-labile enterotoxin (LT).

\section{Results}

Clinical and laboratory features. Most children presented with abdominal symptoms: diarrhoea, abdominal pains, or both (Tables 1 and 2). High fever was characteristic, and 4 infants were admitted because of febrile convulsions. Headache was a common complaint, and one patient was admitted because of a stiff neck. Frank arthritis was not observed, and arthralgia was recorded in only 2

Table 1 Chief complaint at presentation in 40 children with yersiniosis

\begin{tabular}{lc}
\hline Symptoms & No of patients \\
\hline Abdominal symptoms & 29 \\
Febrile convulsions & 4 \\
Headache & 2 \\
Erythema nodosum & 1 \\
Joint pains & 1 \\
Failure to thrive & 1 \\
Bronchitis & 1 \\
Symptomless family contact & 1 \\
\hline
\end{tabular}

Table 2 Clinical manifestations of yersiniosis in 40 children

\begin{tabular}{lll}
\hline Clinical manifestations & \multicolumn{2}{l}{ Patients } \\
\cline { 2 - 3 } & No & $\%$ \\
\hline Fever $\geqslant 38 \cdot 0^{\circ} \mathrm{C}^{*}$ & 36 & 97 \\
Diarrhoea & 32 & 80 \\
mucoid & 16 & \\
watery & 12 & \\
loose stools & 4 & \\
gross blood & 7 & \\
Abdominal pains & 20 & 50 \\
without diarrhoea & 7 & \\
mesenterial lymphadenitis $\dagger$ & 4 & 30 \\
Vomiting & 12 & 20 \\
Headache & 8 & 5 \\
Arthralgia & 2 & 5 \\
Urticaria & 2 & $2 \cdot 5$ \\
Erythema nodosum & 1 & $2 \cdot 5$ \\
Meningeal irritation & 1 & \\
\hline
\end{tabular}

*37 cases recorded. †Appendicectomy performed. cases. One case of erythema nodosum was seen. Blood was cultured in the acute stage of the illness in 8 cases, and in all blood samples the results were negative.

$80 \%$ of the children had diarrhoea. It was more common in the younger age group than in the school-age children, who often had a nondiarrhoeal disease (Fig. 1). Of those with diarrhoea, one half had mucoid stools characteristic of exudative diarrhoea, and in $7(22 \%)$ cases there was gross blood in the stools (Table 2). Faecal leucocytes were found in 4 children out of 5 studied.

Routine laboratory tests frequently showed high erythrocyte sedimentation rate (ESR) and raised white blood cell count (WBC). ESR over $25 \mathrm{~mm}$ in 1st hour was seen in 25 of 36 children (highest 116 in 1st hour), and in those with slower rates the recording was often made only at an early stage of the disease or in late convalescence. The mean WBC was $12.4 \times 10^{9} / 1$ with a range of 5.6 to $30.0 \times 10^{9} / 1$.

The condition of one $2 \frac{1}{2}$-month-old infant was serious, with $15 \%$ dehydration on admission. Five more patients were treated with intravenous fluids for gastroenteritis; the others with diarrhoea were given oral fluids. 32 patients stayed in hospital for a mean period of $6 \cdot 1$ (range 2 to 24) days.

Six children received antibiotics initially because of suspected yersiniosis; one of these had a positive stool culture after the first regimen 1 month later but the culture became negative after a further 2 months. 17 other patients were treated with antibiotics after the results of bacterial cultures had been obtained. Only 5 of the 14 children who did not receive antibiotics were studied bacteriologically at the convalescent stage, and all were negative for $Y$. enterocolitica.

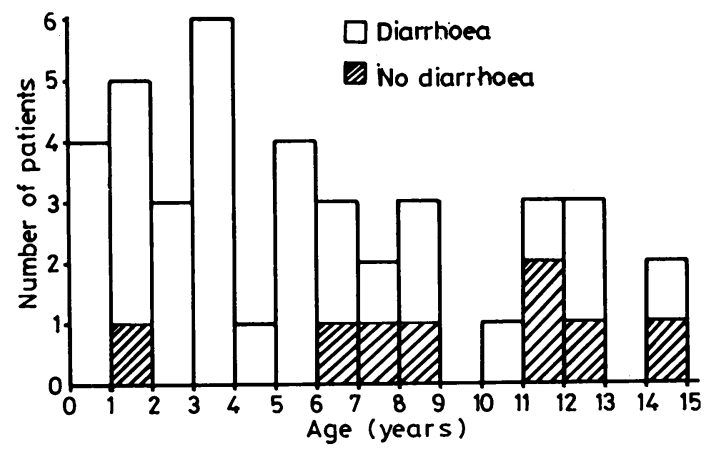

Fig. 1 Age distribution of 40 children with yersiniosis. The case in the age group between 1 and 2 years was a symptomless family contact. 
Bacteriological findings. Of the bacterial isolates 34 were serotype $0: 3,3$ were serotype $0: 9$, and 3 were not typed.

One isolation was made in December 1974, 21 in 1975, 4 in 1976, 9 in 1977, and 5 in 1978. These results reflect not only the probable level of diagnostic activity, but may also show the year-to-year variation in the occurrence of $Y$. enterocolitica infections.

The in vitro sensitivity of 32 of the Y. enterocolitica isolates is presented in Table 3. It is of interest that all the strains were sensitive to sulphonamides and to the combination of sulpha plus trimethoprim (co-trimoxazole). In contrast, practically all strains were resistant to ampicillin and amoxycillin.

30 strains were tested for invasiveness in HEp-2 cells: 29 were found invasive and 1 repeatedly noninvasive. The noninvasive strain belonged to biotype 1 . Of the remaining 29 strains, 5 were of biotype 2 and 24 of biotype 4 . None of the 12 tested strains produced keratoconjunctivitis in the guineapig eye.

Further evidence of the intracellular invasiveness of $Y$. enterocolitica was obtained by electron microscopical examination (Fig. 2). After incubation for 7 hours (standard for the invasiveness test) the bacteria were found located in the cytoplasm, either in single vacuoles or in larger groups, probably resulting from rupture and fusion of several vacuoles. A characteristic feature was the presence of swollen, deformed mitochondria.

29 strains were tested for ST-like toxin production using a newborn mouse assay. A positive toxin reaction was seen in 9 instances. All these strains were also invasive in HEp-2 cells. There appeared to be a correlation between toxin production on reculture and time of isolation: most of the strains that failed to produce toxin were old isolates from 1975 which had been stored in nutrient agar at $4^{\circ} \mathrm{C}$ and recultured at $37^{\circ} \mathrm{C}$ for several years (Table 4). No 'LT-like' enterotoxin activity could be demonstrated in the adrenal cell tests.

Table 3 In vitro sensitivity of 32 strains of $Y$. enterocolitica to selected antibiotics

\begin{tabular}{lccc}
\hline Antibiotics & \multicolumn{3}{l}{ Percentage } \\
\cline { 2 - 4 } & Sensitive & Intermediate & Resistant \\
\hline Gentamicin & 100 & - & - \\
Kanamycin & 100 & - & - \\
Carbenicillin & $12 \cdot 5$ & $40 \cdot 6$ & $46 \cdot 9$ \\
Ampicillin & $6 \cdot 3$ & $3 \cdot 1$ & $90 \cdot 6$ \\
Amoxycillin & $6 \cdot 3$ & - & $93 \cdot 7$ \\
Cephalexin & $34 \cdot 4$ & $50 \cdot 0$ & $15 \cdot 6$ \\
Chloramphenicol & $96 \cdot 9$ & $3 \cdot 1$ & - \\
Tetracycline & 100 & - & - \\
Sulphaisodimidin & 100 & - & - \\
Sulphamethoxazole + trimethoprim & 100 & - & - \\
\hline
\end{tabular}

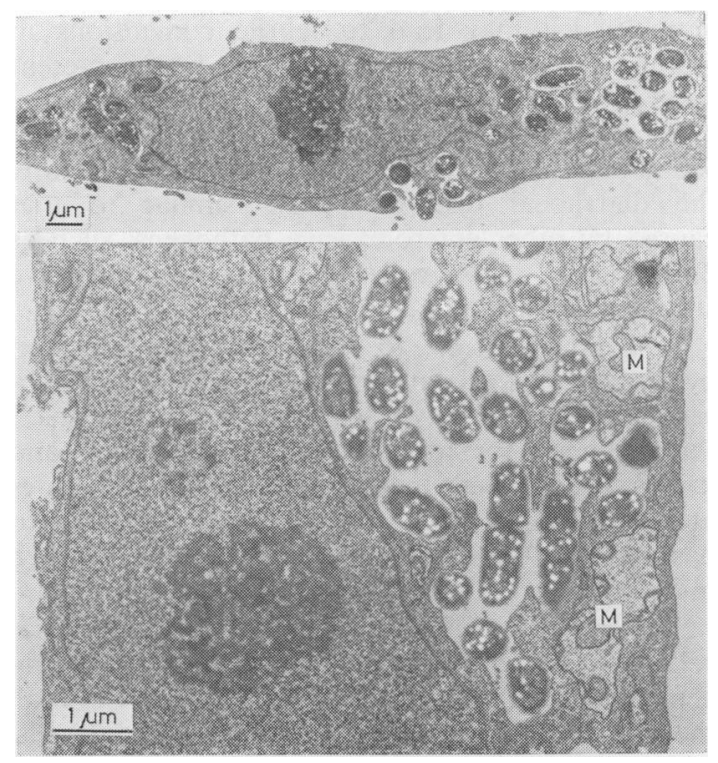

Fig. 2 Electron micrographs of numerous bacteria ( $Y$. enterocolitica) in the cytoplasm of $\mathrm{HEp}-2$ cells. The bacteria are located in vacuoles separately or in groups (top). Swollen deformed mitochondria $(M)$ can be seen (bottom).

Table 4 Results of heat-stable enterotoxin assay in newborn mice of 29 strains of $Y$. enterocolitica according to the year of isolation

\begin{tabular}{llllllll}
\hline Baby mouse assay & \multicolumn{3}{l}{ Year of isolation } & \multirow{2}{*}{ Total } \\
\cline { 2 - 7 } & 74 & 75 & 76 & 77 & 78 & \\
\hline Positive & - & 2 & 2 & 2 & 3 & 9 \\
Negative & 1 & 16 & 1 & 1 & 1 & 20 \\
\hline
\end{tabular}

\section{Discussion}

Many clinical features of $Y$. enterocolitica infections appear consistent with the concept of an invasive pathogenic mechanism. Frank blood stream infections by $Y$. enterocolitica are rare and may be associated with some underlying condition affecting host defence mechanisms. ${ }^{19-20}$ More often the infection is confined to mesenteric lymph nodes with resultant lymphadenitis and acute appendicitislike disease. The most common form of $Y$. enterocolitica infections in childhood is gastroenteritis, which because of its apparent mildness may not at first seem to fit into the same picture of invasive infection. However, these data show that gastroenteritis is often associated with signs of systemic infection - that is high WBC, high ESR, and high 
fever-which may lead to febrile convulsions in infants. Furthermore, the diarrhoea in the present series was clinically exudative in at least half the patients, and polymorphonuclear leucocytes were found in the stools in $80 \%$ of the children. Therefore, the diarrhoeal disease appears similar to that caused by other enteroinvasive pathogens, such as Shigella sp., Salmonella sp., and enteroinvasive strains of Escherichia coli. ${ }^{21}$

It is now possible to induce invasive infection by $Y$. enterocolitica in laboratory mice with a subsequent disease closely resembling that in humans. ${ }^{22}$ Pathogenicity for mice is strain-dependent, ${ }^{23}$ and it has been noted that $Y$. enterocolitica isolates attenuate easily, so that it may be necessary to passage isolates from culture collections through mice before production of typical diseases. ${ }^{22}$

The in vitro invasiveness test in human epithelial cells may also constitute a relevant model for pathogenic studies of $Y$. enterocolitica. We found that all the human isolates were invasive for HEp-2 cells, with one exception. The one exception represented a different biotype (type 1) from all the other human faecal isolates (biotypes 2 or 4). Our observations are in accordance with those of Une et al. ${ }^{24}$ who also found human patient isolates (serotypes $0: 3$, $0: 5 \mathrm{~B}, 0: 8$, and $0: 9$ ) invasive for HeLa cells in vitro, whereas strains from healthy humans and various animals were found noninvasive. Similarly, Lee et al. ${ }^{7}$ found typical clinical strains to be invasive for $\mathrm{HeLa}$ cells. They also stated that in vitro invasiveness is a stable property not lost in stock cultures. Recently, Pedersen et al. reported lack of invasiveness in HeLa cells of $Y$. enterocolitica strains belonging to biotype $1 .{ }^{25}$

All the tested strains of $Y$. enterocolitica failed to produce keratoconjuctivitis in the guinea-pig eye. This is in accordance with the report of Pai and Mors. ${ }^{16}$ It appears that cultured human epithelial cells may be more sensitive than the Serény test for the detection of intracellular invasiveness, at least for $Y$. enterocolitica. Enterotoxin production has been recently advanced as an alternative pathogenic mechanism of $Y$. enterocolitica, which could play a role in inducing watery diarrhoea in some children. ${ }^{16}$ However, Pai and Mors demonstrated that enterotoxin was produced only at temperatures below $30^{\circ} \mathrm{C}$ and therefore questioned its role in vivo. They further demonstrated toxin production by serotypes not considered pathogenic in humans. ${ }^{26}$ We were also able to detect this enterotoxin, which resembles the ST enterotoxin of $E$. coli, by the newborn mouse assay. In our hands, however, only some strains of $Y$. enterocolitica were enterotoxigenic, and the property of toxin production appeared to be lost in prolonged stock culture. Nevertheless, we do not wish to abandon the concept of enterotoxigenicity of $Y$. enterocolitica as a possible pathogenic mechanism in yersiniosis. At least two possibilities remain: first, a preformed toxin may act on epithelial cells to induce diarrhoea. This hypothesis should in any case be tested. The second possibility is that toxin production, ST-like or some other, might be an auxiliary pathogenic mechanism acting intracellularly. We propose that the swollen deformed mitochondria found in the epithelial cells invaded by $Y$. enterocolitica could be attributed to a toxinlike mechanism. It was suggested that a toxic metabolite produced intracellularly by invasive $E$. coli could destroy epithelial cells and thus lead to colitis and dysentery. ${ }^{27}$

In conclusion, we suggest that bacterial properties in the pathogenesis of $Y$. enterocolitica infections include both invasiveness and enterotoxin production. The invasive ability is associated both with the serotype and the biotype. In future studies the toxigenicity should be considered as an additional mechanism, not only in the induction of diarrhoea but also in systemic disease.

In most cases $Y$. enterocolitica infections in children appear to be self-limiting, despite the invasiveness of the pathogen. Antibiotic treatment is therefore seldom necessary. However, when it is administered on clinical grounds, the results of in vitro antibiotic sensitivity testing should be considered. In our experience, and in that of others, ${ }^{28}$ sulpha plus trimethoprim (co-trimoxazole) currently appears to be the drug of choice in the treatment of $Y$. enterocolitica infections both in adults and children.

We thank Miss Kerttu Saarinen, Mrs Taimi Tammipuu, and Miss Kerttu Kangas for technical assistance.

The study was supported by grants from the National Medical Council, the Academy of Finland, the Emil Aaltonen Foundation, and the Finnish Foundation for Paediatric Research.

\section{References}

1 Ahvonen P. Human yersiniosis in Finland. II. Clinical features. Ann Clin Res 1972; 4: 39-48.

2 Arvastson B, Damgaard K, Winblad S. Clinical symptoms of infection with Yersinia enterocolitica. Scand J Infect Dis 1971; 3: 37-40.

3 Leino R, Kalliomäki J L. Yersiniosis as an internal disease. Ann Intern Med 1974; 81: 458-61.

4 Bergstrand C G, Winblad S. Clinical manifestations of infection with Yersinia enterocolitica in children. Acta Paediatr Scand 1974; 63: 875-7. 
5 Delorme J, Laverdière $\mathrm{M}$, Martineau $\mathrm{B}$, Lafleur L. Yersiniosis in children. Can Med Assoc J 1974; 110: 281-4.

6 Jepsen O B, Korner B, Lauritsen K B, et al. Yersinia enterocolitica infections in patients with acute surgical abdominal disease. Scand J Infect Dis 1976; 8: 189-94.

7 Lee W H, McGrath P P, Carter P H, Eide E L. The ability of some Yersinia enterocolitica strains to invade HeLa cells. Can J Microbiol 1977; 23: 1714-22.

8 Une T. Studies on the pathogenicity of Yersinia enterocolitica. II. Interaction with cultured cells in vitro. Microbiol Immunol 1977; 21 : 365-77.

9 Mäki M, Grönroos $\mathrm{P}$, Vesikari T. In vitro invasiveness of Yersinia enterocolitica isolated from children with diarrhea. J Infect Dis 1978; 138: 677-80.

10 Wauters G. Improved methods for the isolation and recognition of Yersinia enterocolitica. In: Winblad S, ed. Yersinia, Pasteurella, and Francisella. Contributions to microbiology and immunology. Vol. 2. Basel: Karger, 1972: 68-70.

11 Sonnenwirth A C. Yersinia. In: Lennette E H, Spaulding E H, Truant J P, eds. Manual of clinical microbiology, second edition. Washington DC: American Society for Microbiology, 1974: 222-9.

12 Ahvonen P. Human yersiniosis in Finland. I. Bacteriology and serology. Ann Clin Res 1972; 4: 30-8.

13 Ericsson H M, Sherris J C. Antibiotic sensitivity testing. Report of an international collaborative study. Acta Pathol Microbiol Scand (B) 1971; Supplement 217.

14 Alestig K, Dornbusch K, Ericsson C, et al. Resistance determination of bacteria. A new classification in sensitivity groups-S, I, and R. Läkartidningen 1978; 75: 4346-8.

15 Serény B. Experimental shigella keratoconjunctivitis: a preliminary report. Acta Microbiol Acad Sci Hung $1955 ; 2: 293-6$.

${ }_{16}$ Pai C H, Mors V. Production of enterotoxin by Yersinia enterocolitica. Infect Immun 1978; 19: 908-11.

17 Dean A G, Ching Y-C, Williams R G, Harden L B. Test for Escherichia coli enterotoxin using infant mice: application in a study of diarrhea in children in Honolulu. J Infect Dis 1972; 125: 407-11.
18 Donta S T, Moon H W, Whipp S C. Detection of heatlabile Escherichia coli enterotoxin with the use of adrenal cells in tissue culture. Science $1974 ;$ 183: 334-5.

19 Reinicke V, Korner B. Fulminant septicaemia caused by Yersinia enterocolitica. Scand J Infect Dis 1977; 9: 249-51.

20 Butzler J P, Alexander M, Segers A, Cremer N, Blum D. Enteritis abscess, and septicemia due to Yersinia enterocolitica in a child with thalassemia. J Pediatr 1978; 93: 619-21.

21 Pickering L K, DuPont H, Olarte J, Conklin R, Ericsson C. Fecal leukocytes in enteric infections. $A m \mathrm{~J}$ Clin Pathol 1977; 68: 562-5.

22 Carter P B. Pathogenicity of Yersinia enterocolitica for mice. Infect Immun 1975; 11 : 164-70.

${ }^{23}$ Quan T J, Meek J L, Tsuchiya K R, Hudson B W, Barnes A M. Experimental pathogenicity of recent North American isolates of Yersinia enterocolitica. J Infect Dis 1974; 129: 341-4.

24 Une T, Zen-Yoji H, Maruyama T, Yanagawa Y. Correlations between epithelial cell infectivity in vitro and O-antigen groups of Yersinia enterocolitica. Microbiol Immunol 1977; 21: 727-9.

25 Pedersen K B, Winblad S, Bitsch V. Studies on the interaction between different $\mathrm{O}$-serotypes of Yersinia enterocolitica and HeLa cells. Acta Pathol Microbiol Scand (B) 1979; 87B: 141-5.

26 Pai C H, Mors V, Toma S. Prevalence of enterotoxigenicity in human and nonhuman isolates of Yersinia enterocolitica. Infect Immun 1978; 22: 334-8.

27 Moon H W, Isaacson R E, Pohlenz J. Mechanisms of association of enteropathogenic Escherichia coli with intestinal epithelium. Am J Clin Nutr 1979; 32: 119-27.

28 Hammerberg S, Sorger S, Marks M I. Antimicrobial susceptibilities of Yersinia enterocolitica biotype 4 serotype O:3. Antimicrob Agents Chemother 1977; 11: 566-8.

Correspondence to Dr Markku Mäki, Institute of Clinical Sciences, University of Tampere, PO Box 607, SF-33101 Tampere 10, Finland.

Received 26 September 1979 